\title{
Análise de mecânicas de jogos e tendências gráficas na representação de jogos de procedimentos médicos, visando o estímulo e motivação para o aprendizado \\ Analysis of game mechanics and graphical trends in the representation of games of medical procedures, aiming at the stimulus and motivation for learning
}

Daniella Rosito Michelena Munhoz, Larissa Ugaya Mazza, Marcelo Satoshi Taguchi, Carla Galvão Spinillo, Luciane Fadel, Ana Emília Figueiredo de Oliveira, Ana Estela Haddad, Dilson José Lins Rabêlo Júnior \& Alan Palomero Machado Goes Rodrigues

design de jogos, jogos para a saúde, jogos para a educação, jogos sérios

\begin{abstract}
Diante do potencial de utilizar sistemas de jogo no ensino, tem início um projeto para o desenvolvimento de um jogo sério de simulação de casos clínicos em uma parceria entre a UFPR, UNA-SUS/UFMA e USP. A primeira fase deste projeto consiste na análise de jogos que lidam com procedimento médicos. Para essa análise foi elaborado um modelo baseado no protocolo de Munhoz (2018) para jogos cooperativos e adaptado para jogos de saúde. A amostra foi coletada por conveniência por 3 fontes diferente (sites de jogos online, plataformas de compra de jogos e sites de recomendação de jogo) e totalizou em 10 jogos, todos com o tema medicina, sendo, 8 para entretenimento e 2 para aprendizagem. Os resultados mostraram características dos jogos encontrados no mercado no sentido de abrir possibilidades para desenvolver sistemas de jogos a partir das lacunas observadas.
\end{abstract}

game design, games for health, games for education, serious games

Faced with the potential of using gaming systems in education, a project for the development of a serious simulation game of clinical cases in a partnership between UFPR, UNA-SUS / UFMA and USP is beginning. The first phase of this project consists of analyzing games that deal with medical procedure. For this analysis, a model based on the Munhoz protocol (2018) was developed for cooperative games and adapted for health games. The sample was collected for convenience by 3 different sources (online gaming sites, gaming platforms and game recommendation sites) and totaled 10 games, all with the theme medicine, 8 for entertainment and 2 for learning. The results showed that characteristics of the games found on the market opening the possibility of developing game systems from the gaps observed.

\section{Introdução e objetivo}

A inclusão de sistemas de jogos como instrumento para educação pode vir a engajar os estudantes, ajudar na retenção de memória de curto prazo, no reconhecimento de padrões e coordenação (Michael \& Chen, 2006). Um desses instrumentos são os jogos sérios. Esses jogos são construídos com o objetivo de ensinar e não tem como foco principal o entretenimento, o que não significa que eles não possam conter aspectos lúdicos (Susi et al. 2015; Michael \& Chen, 2006).

Jogos com propósito educativo articulam os elementos de jogo para tornar o ensino mais lúdico e, possivelmente, engajante (Rodriguez, 2006; Garris et al. 2002). O uso de jogos no ensino estimula a exploração devido a sua alta interação, o que pode levar ao fenômeno de aprendizado pela descoberta (Betrancourt, 2005), que acontece quando os alunos chegam às suas próprias conclusões depois de conhecer os fatos. Os jogos sérios também podem ser utilizados como um recurso de ensino a distância (Rodriguez, 2006), e podem estimular funções psicológicas secundária que servem para a conscientização e preparação dos jogadores para novas atividades (Munhoz, 2018).

Anais do 9 CIDI e 9 CONGIC

Luciane Maria Fadel, Carla Spinillo, Anderson Horta, Cristina Portugal (orgs.)

Sociedade Brasileira de Design da Informação - SBDI Belo Horizonte | Brasil | 2019

ISBN $978-85-212-1728-2$
Proceedings of the 9th CIDI and 9th CONGIC Luciane Maria Fadel, Carla Spinillo, Anderson Horta, Cristina Portugal (orgs.)

Sociedade Brasileira de Design da Informação - SBDI Belo Horizonte | Brazil | 2019

ISBN 978-85-212-1728-2 
Munhoz, D. R. M., Mazza, L. U., Taguchi, M. S., Spinillo, C. G., Fadel, L. M., Oliveira, A. E. F., Haddad, A. E., Rabêlo Jr., D. J. L. \& Rodrigues, A. P. M. G. | Análise de mecânicas de jogos e tendências gráficas na representação de jogos de procedimentos médicos, visando o estímulo e motivação para o aprendizado

O desenvolvimento de jogos para o aprendizado difere do desenvolvimento dos jogos para entretenimento (Susi et al. 2015). Quando se desenvolve um jogo para o aprendizado o foco deve estar na solução de problemas e na situação de aprendizado. A simulação deve ser elaborada em conformidade com o conteúdo da aprendizagem e a comunicação não precisa ser completa (Susi et al. 2015) deixando espaço para o aluno desenvolver suas habilidades. Quando bem desenvolvido, um jogo tem a capacidade de engajar o jogador no Ciclo do Jogo (Garris et al. 2002). O Ciclo do Jogo acontece quando o jogo motiva o jogador a persistir, superar os desafios e ficar engajado à atividade. Esse efeito apenas acontece se jogadores se sentirem motivados o suficiente para constantemente retornar ao jogo (Garris et al, 2002).

Jogos sérios já foram utilizados no passado com resultados positivos (Castel et al. 2018; Willmott et al. 2018; Shepard et al. 2018; Barnes \& Prescott, 2018), o que mostra o possível benefício em adotar um jogo para o ensino ou treinamento. Por outro lado, encontramos no mercado jogos com temas sérios (e.g. procedimentos médicos) projetados unicamente para o entretenimento, sem embasamento técnico-científico. Assim, temos contradições entre jogos do mercado e a literatura sobre jogos sérios e design de sistemas de informação.

Diante dessa lacuna, vimos o potencial de desenvolver um jogo sério de simulação casos clínicos para a UNA-SUS/UFMA. Esta foi criada no final de 2009, quando a Universidade Federal do Maranhão (UFMA) adere à rede UNA-SUS (Universidade Aberta do SUS). A instituição começou as atividades em 2010 e oferece cursos de especialização e materiais de apoio para profissionais de medicina, utilizando recursos de ensino a distância. Dentre estes recursos tem-se o uso de jogos educativos em saúde. Todavia, o desenvolvimento destes jogos tem se dado de forma tácita pelas equipes da UNA-SUS/UFMA (e.g., pedagogos, especialistas em TI-tecnologia da Informação, designers gráficos), sem os expertises de game designers e designers da informação. Considerando a importância destes expertises para a gamificação de conteúdos em saúde, iniciou-se um projeto de pesquisa aplicada para o desenvolvimento de jogos sérios pela UNA-SUS/UFMA. Para isto, foi realizado um estudo analítico de uma amostra de jogos na área de medicina, a fim de caracterizar como conteúdos procedimentais médicos estão sendo gamificados, o qual é o foco deste artigo.

\section{Estudo analítico de jogos procedimentais na área médica}

O estudo teve como objetivo identificar as características da representacionais dos jogos de procedimentos médicos sob uma perspectiva do design de jogos e do design de sistemas de informação. Para isto, foi adaptado o modelo de análise de jogos cooperativos de Munhoz (2018). O modelo adaptado (ver item 3 deste artigo) combinou elementos de jogo e de representação gráfica para descrição dos componentes da estética, mecânica e dinâmica (HUNICKE et al, 2004) da amostra.

Amostra: foi coletada uma amostra por conveniência de 10 jogos procedimentais de medicina. em sites de entretenimento (e.g. ojogos.com.br), plataformas de compra de jogos (e.g. Steam) e sites de crítica de jogos (e.g. IGN e Board Game Geek). A análise foi realizada nos seguintes jogos:

- Amatour surgeon. By: Cartoon Network. Publisher: Adult Swim (2008);

- Doctor Wars. Publisher: Lurp Designs LLC (2012);

- Healthy Heart Hospital. Publisher: Victory Point Games (2015);

- Life and Death. By: Software Toolworks. Publisher: Software Toolworks, Mindscape, Ving Co., Ltd., Takeru (1988);

- LifeSign: Surgical Unit. By: Spike. Publisher: Spike, JoWooD Entertainment, DreamCatcher Interactive (2005);

- Operate now! Publisher: Spil Games (2016); 
Munhoz, D. R. M., Mazza, L. U., Taguchi, M. S., Spinillo, C. G., Fadel, L. M., Oliveira, A. E. F., Haddad, A. E., Rabêlo Jr., D. J. L. \& Rodrigues, A. P. M. G. | Análise de mecânicas de jogos e tendências gráficas na representação de jogos de procedimentos médicos, visando o estímulo e motivação para o aprendizado

- Occam's Razor: The Diagnostician's Dilemma. By: Nerdcore Medical (2013)

- Prognosis: Your diagnosis. By: Medical Joyworks;

- Surgeon simulator. By: Bossa Studios (2013);

- Trauma Center: Under the Knife. By: Atlus (2005).

Procedimentos e forma de análise dos resultados: A análise se deu pela interação, observação e leitura das regras dos jogos selecionados. Os jogos de plataforma e aplicativos foram jogados, nos analógicos foram lidas as regras e os de console foram assistidos gameplays no Youtube. Os resultados foram analisados de forma qualitativa.

\section{Modelo de análise}

O modelo de análise tem base no framework desenvolvido por Munhoz (2018) no estudo sobre o design de jogos de tabuleiro e dinâmicas cooperativas. Este modelo compreende o jogo como um sistema, ou seja, um conjunto de elementos que interagem e se relacionam formando um todo complexo (Salen \& Zimmerman, 2004). O framework original especifica elementos de jogo no sentido de identificar a dinâmica cooperativa em jogos. Para o presente trabalho ocorre uma adaptação do modelo visando analisar jogos com a temática da medicina, mais especificamente procedimentos médicos. O framework é constituído por aspectos conceituais que vão orientar a estética do jogo e aspectos operacionais que vão estabelecer as mecânicas do jogo pela perspectiva do MDA (Mechanics, Dynamics, Aesthetic) (Hunicke et al, 2004). A definição destes elementos visa promover uma dinâmica de jogo que leva o jogador a uma experiência que emerge da atividade de jogar.

O modelo distingue aspectos conceituais (tabela 1), aspectos operacionais (tabela 2) do jogo e em seguida sua adaptação (tabela 3) para análise de jogos de temática médica.

Tabela 1: Aspectos conceituais - As determinações estabelecidas para o jogo (usado com permissão de Munhoz)

\begin{tabular}{|c|c|c|}
\hline $\begin{array}{l}\text { Motivo } \\
\text { - Sobre o que é o jogo. }\end{array}$ & $\begin{array}{l}\text { Desafio - O que os jogadores } \\
\text { vão enfrentar? }\end{array}$ & $\begin{array}{l}\text { Interação - Entre jogadores e entre } \\
\text { os jogadores e o sistema de jogo. }\end{array}$ \\
\hline \multirow{3}{*}{$\begin{array}{l}\text { Tema / Situação imaginária: } \\
\text { aventura e heroísmo; } \\
\text { sobrevivência e fuga; } \\
\text { estratégia e poder; } \\
\text { administração e política; } \\
\text { economia e comércio; } \\
\text { construção e produção. }\end{array}$} & $\begin{array}{l}\text { Conflito: jogadores vs jogo; time } \\
\text { vs time. Foco em um oponente; } \\
\text { foco em muitos oponentes. }\end{array}$ & $\begin{array}{l}\text { Comunicação entre os jogadores: } \\
\text { livre; limitada. } \\
\text { Implícita; explícita. }\end{array}$ \\
\hline & $\begin{array}{l}\text { Objetivo: comum; individual. } \\
\text { Fixo; variável. }\end{array}$ & \multirow{2}{*}{$\begin{array}{l}\text { Representação do jogador: } \\
\text { Personagem; território; outro. } \\
\text { Habilidades iguais; diferentes. } \\
\text { Controla um personagem; vários. }\end{array}$} \\
\hline & $\begin{array}{l}\text { Ameaças: ativas; passivas. } \\
\text { Naturais; artificiais; fantásticas. }\end{array}$ & \\
\hline
\end{tabular}

A tabela 1 apresenta os aspectos conceituais do jogo, divididos em:

- motivo / situação imaginária - os jogos em geral são idealizados a partir de temas que visam motivar os jogadores a experimentar uma situação imaginária simulada em ambiente seguro no sentido de promover diferentes experiências aos jogadores. Originalmente o framework apresenta temas motivadores para jogos cooperativos como: heroísmo ou sobrevivência. Temas que, nos jogos cooperativos, levam os jogadores a situações imaginárias que estimulam a cooperação. O tema oferece a diegese do jogo, a dimensão narrativa, uma 'realidade ficcional' composta por cenários, personagens e situação fictícia que ambienta a experiência de jogo gerando uma situação imaginário que vai promover estesis, estimular funções cognitivas e psicológicas no jogador (Munhoz, 2018);

- desafio - o desafio é um fator essencial em jogos. No framework o desafio é compreendido pelo conflito, objetivo e ameaças. O conflito se dá pelo arranjo entre 
Munhoz, D. R. M., Mazza, L. U., Taguchi, M. S., Spinillo, C. G., Fadel, L. M., Oliveira, A. E. F., Haddad, A. E., Rabêlo Jr., D. J. L. \& Rodrigues, A. P. M. G. | Análise de mecânicas de jogos e tendências gráficas na representação de jogos de procedimentos médicos, visando o estímulo e motivação para o aprendizado

jogador(es) e o sistema de jogo, podendo o jogador estar em oposição ao sistema (single player) ou em oposição a outros jogadores (multiplayer). O objetivo pode ser único - o mesmo para todos os jogadores, ou pode ser individualizado - objetivos diferentes para cada jogador. As ameaças são elementos importantes para elevar o grau de dificuldade em um jogo, ampliar a dinâmica de jogo e deixar a experiência mais estimulante;

- interação - a interação compreende tanto a interação dos jogadores com o sistema de jogo (single player), bem como a interação e a comunicação entre os jogadores no caso de jogos multiplayers. Compreende também como o jogador é representado no jogo, se performatiza um personagem (e.g. avatar) ou administra uma equipe, ou tem o controle total sobre o ambiente de jogo (e.g. god game).

Tabela 2: Aspectos operacionais - Os mecanismos projetados para progressão do jogo (usado com permissão de Munhoz).

\begin{tabular}{l|l|l}
$\begin{array}{l}\text { Progressão do jogo } \\
\text { Alternância de turnos }\end{array}$ & $\begin{array}{l}\text { Turno do jogador - Como } \\
\text { ocorre o turno dos jogadores? }\end{array}$ & $\begin{array}{l}\text { Turno do sistema - Como ocorre o } \\
\text { turno do jogo? }\end{array}$ \\
\hline $\begin{array}{l}\text { Estado do jogo: ordem das } \\
\text { fases, situação } \\
\text { momento-a-momento, } \\
\text { análise parálise }\end{array}$ & $\begin{array}{l}\text { Ações do jogador } \\
\text { (mecânicas): físicas; de } \\
\text { economia; de progressão; } \\
\text { táticas; de interação social }\end{array}$ & $\begin{array}{l}\text { Sistema do jogo: ações e eventos do } \\
\text { sistema de jogo transmitidas pelas } \\
\text { regras do jogo }\end{array}$ \\
\hline $\begin{array}{l}\text { Informação sobre o jogo: } \\
\text { feedback (O que?, Onde?) }\end{array}$ & $\begin{array}{l}\text { Compartilhamento de itens, } \\
\text { informações, outros: } \\
\text { compartilhar; não compartilhar }\end{array}$ & $\begin{array}{l}\text { Risco e recompensa: ameaças fixas; } \\
\text { ameaças aumentam (constantemente, } \\
\text { randomicamente ou } \\
\text { exponencialmente) }\end{array}$ \\
\hline
\end{tabular}

A tabela 2 apresenta os aspectos operacionais do jogo, divididos em:

- progressão do jogo - o jogo é um sistema dinâmico que se desenvolve momento-amomento em uma sequência de eventos e ações. A dinâmica do jogo ocorre pela contínua interação do(s) jogador(es) com o sistema de jogo. Em um momento o sistema de jogo apresenta um desafio ao jogador e fica em 'modo de espera' (stand by) aguardando uma ação do jogador para que ocorra a progressão do jogo. No modo de espera o jogador deve realizar uma ação para o jogo avançar. O modo de espera é chamado de estado do jogo, sendo este o momento em que o sistema apresenta um leque de possibilidades ao jogador e fica parado até que o jogador faça uma escolha (tomada de decisão) e realize sua ação. O tempo que o jogador ocupa decidindo o que fazer, particularmente quando é muito demorado, é chamado de análise paralise. Em cada momento (estado do jogo) o sistema de jogo oferece informações (feedback) ao jogador. São informações sobre o leque de opções oferecido pelo sistema, bem como as informações sobre a situação e o desempenho do jogador durante o jogo. Esta atividade desenvolvida momento-a-momento permite que o jogador tire suas próprias conclusões depois de conhecer os fatos e tenha um espaço para desenvolver suas habilidades motoras, cognitivas e/ou psicológicas;

- turno do jogador - a fase do jogador compreende as ações realizadas pelos jogadores. As ações disponíveis no jogo foram classificadas por Dormans (2012) em mecânicas físicas, econômicas, de progressão, táticas ou de interação social;

- turno do sistema - as ações ou eventos do sistema de jogo seguem instruções predeterminadas no projeto do jogo (inteligência artificial). Nos jogos de oposição entre os jogadores (multiplayer) a própria interação entre os jogadores estabelece um equilíbrio de forças, um jogador é obstáculo para o jogador adversário. Nos jogos em que jogador joga diretamente com o sistema (single player) é necessário que o sistema reaja à ação do jogador. Um projeto do jogo que contemple um leque de possibilidades mais abrangente permite uma experiência mais desafiadora ao jogador. Riscos e recompensas são elementos importantes para elevar o grau de dificuldade em um jogo 
Munhoz, D. R. M., Mazza, L. U., Taguchi, M. S., Spinillo, C. G., Fadel, L. M., Oliveira, A. E. F., Haddad, A. E., Rabêlo Jr., D. J. L. \& Rodrigues, A. P. M. G. | Análise de mecânicas de jogos e tendências gráficas na representação de jogos de procedimentos médicos, visando o estímulo e motivação para o aprendizado

e deixar a experiência mais estimulante e desafiadora, o que deve motivar o jogador a persistir, superar os desafios e ficar engajado à atividade.

\section{Adaptação do modelo}

As tabelas 3 e 4 apresentam a adaptação do framework original para o modelo de análise objeto deste estudo.

Tabela 3: Adaptação do modelo de análise. Aspectos conceituais

\begin{tabular}{|c|c|c|c|}
\hline \multicolumn{2}{|c|}{ Aspectos conceituais } & \multirow[b]{2}{*}{$\begin{array}{l}\text { Original } \\
\text { aventura e heroísmo; } \\
\text { sobrevivência e fuga; } \\
\text { estratégia e poder, } \\
\text { administração e política; } \\
\text { economia e comércio; } \\
\text { construção e produção }\end{array}$} & \multirow{2}{*}{$\begin{array}{l}\text { Adaptado } \\
\text { simulador de cirurgia; } \\
\text { gestão hospitalar; } \\
\text { atendimento clínico }\end{array}$} \\
\hline Motivo / Motivação & $\begin{array}{l}\text { Tema / Situação } \\
\text { imaginária }\end{array}$ & & \\
\hline & Propósito & $\begin{array}{l}\text { não foi contemplado no } \\
\text { framework original }\end{array}$ & $\begin{array}{l}\text { entretenimento; } \\
\text { aprendizagem }\end{array}$ \\
\hline \multirow[t]{3}{*}{ Desafio } & Conflito & $\begin{array}{l}\text { jogadores vs jogo; } \\
\text { time vs time } \\
\text { foco em um oponente; } \\
\text { foco em muitos oponentes }\end{array}$ & $\begin{array}{l}\text { Player/s VS Game; } \\
\text { Player VS Player }\end{array}$ \\
\hline & Objetivo & $\begin{array}{l}\text { comum; individual } \\
\text { fixo; variável }\end{array}$ & $\begin{array}{l}\text { saúde ou cura; } \\
\text { gestão; } \\
\text { zombar }\end{array}$ \\
\hline & Ameaças & $\begin{array}{l}\text { ativas; passivas } \\
\text { naturais; artificiais; } \\
\text { fantásticas }\end{array}$ & $\begin{array}{l}\text { ao avatar do jogador; } \\
\text { ao paciente; } \\
\text { situacional }\end{array}$ \\
\hline \multirow[t]{2}{*}{ Interação } & $\begin{array}{l}\text { Comunicação entre os } \\
\text { jogadores }\end{array}$ & $\begin{array}{l}\text { livre; limitada } \\
\text { implícita; explícita. }\end{array}$ & Não se aplica \\
\hline & $\begin{array}{l}\text { Representação do } \\
\text { jogador }\end{array}$ & $\begin{array}{l}\text { Personagem; território; } \\
\text { habilidades iguais; } \\
\text { habilidades diferentes } \\
\text { controlar um personagem; } \\
\text { controlar vários }\end{array}$ & $\begin{array}{l}\text { avatar fixo; } \\
\text { avatar customizável } \\
\text { habilidades iguais; } \\
\text { habilidades diferentes. } \\
\text { habilidades fixas; } \\
\text { habilidades crescentes }\end{array}$ \\
\hline \multicolumn{2}{|c|}{$\begin{array}{l}\text { Tabela 4: Adaptação do modelo de análise. Aspectos } \\
\text { Aspectos operacionais }\end{array}$} & $\begin{array}{l}\text { peracionais } \\
\text { Original }\end{array}$ & Adaptado \\
\hline \multirow[t]{5}{*}{$\begin{array}{l}\text { Progressão / } \\
\text { Processo do jogo }\end{array}$} & Estado do jogo & turnos / ordem das fases & $\begin{array}{l}\text { mundo aberto; } \\
\text { mundo fechado }\end{array}$ \\
\hline & $\begin{array}{l}\text { Análise parálise / } \\
\text { Estado de espera nas } \\
\text { pausas }\end{array}$ & $\begin{array}{l}\text { ocorre; } \\
\text { não ocorre }\end{array}$ & $\begin{array}{l}\text { congelado; idle; } \\
\text { timer, advertência }\end{array}$ \\
\hline & $\begin{array}{l}\text { Informação do jogo / } \\
\text { feedback }\end{array}$ & O que? & $\begin{array}{l}\text { personagem; ameaça; } \\
\text { tempo; situação }\end{array}$ \\
\hline & Posição do feedback & Onde? & $\begin{array}{l}\text { superior; inferior } \\
\text { direita; esquerda; centro }\end{array}$ \\
\hline & Feedback pedagógico & $\begin{array}{l}\text { não foi contemplado no } \\
\text { framework original }\end{array}$ & $\begin{array}{l}\text { consecutivo; } \\
\text { final }\end{array}$ \\
\hline \multirow[t]{2}{*}{ Turno do jogador } & $\begin{array}{l}\text { Ações do jogador } \\
\text { (mecânicas) }\end{array}$ & $\begin{array}{l}\text { físicas; } \\
\text { de economia; } \\
\text { de progressão; } \\
\text { táticas; } \\
\text { de interação social } \\
\end{array}$ & $\begin{array}{l}\text { físicas; } \\
\text { de economia; } \\
\text { de progressão; } \\
\text { táticas; } \\
\text { de interação social } \\
\end{array}$ \\
\hline & $\begin{array}{l}\text { Compartilhamento de } \\
\text { itens / Objetos } \\
\text { manipuláveis }\end{array}$ & $\begin{array}{l}\text { compartilhar; } \\
\text { não compartilhar }\end{array}$ & $\begin{array}{l}\text { paciente; } \\
\text { props }\end{array}$ \\
\hline $\begin{array}{l}\text { Turno do sistema / } \\
\text { Eventos do sistema }\end{array}$ & $\begin{array}{l}\text { IA - Inteligência } \\
\text { artificial }\end{array}$ & $\begin{array}{l}\text { Sistema do jogo (ações e } \\
\text { eventos do sistema de }\end{array}$ & $\begin{array}{l}\text { randomizada; } \\
\text { progressão situacional }\end{array}$ \\
\hline
\end{tabular}

Anais do 9 Congresso Internacional de Design da Informação | CIDI 2019 
Munhoz, D. R. M., Mazza, L. U., Taguchi, M. S., Spinillo, C. G., Fadel, L. M., Oliveira, A. E. F., Haddad, A. E., Rabêlo Jr., D. J. L. \& Rodrigues, A. P. M. G. | Análise de mecânicas de jogos e tendências gráficas na representação de jogos de procedimentos médicos, visando o estímulo e motivação para o aprendizado

\begin{tabular}{|c|c|c|c|}
\hline & & $\begin{array}{l}\text { jogo) transmitidas pelas } \\
\text { regras do jogo }\end{array}$ & \\
\hline & Risco e recompensa & $\begin{array}{l}\text { ameaças fixas; } \\
\text { ameaças aumentam } \\
\text { (constantemente, } \\
\text { randomicamente ou } \\
\text { exponencialmente) }\end{array}$ & $\begin{array}{l}\text { riscos fixos; } \\
\text { riscos crescentes. } \\
\text { recompensa (monetária, } \\
\text { progressão, bônus) }\end{array}$ \\
\hline \multirow[t]{2}{*}{ Meio } & Plataforma & $\begin{array}{l}\text { não foi contemplado no } \\
\text { framework original }\end{array}$ & $\begin{array}{l}\text { celular; } \\
\text { computador; } \\
\text { console; } \\
\text { analógico }\end{array}$ \\
\hline & Recursos & $\begin{array}{l}\text { não foi contemplado no } \\
\text { framework original }\end{array}$ & $\begin{array}{l}\text { RV - realidade virtual } \\
\text { RA - realidade aumentada }\end{array}$ \\
\hline
\end{tabular}

Para o presente trabalho ocorreram as seguintes adaptações do modelo.

- motivação - o motivo é baseado no tema maior da medicina e nas situações imaginárias de procedimentos médicos identificados pela simulação de cirurgia, gestão hospitalar e atendimento clínico. Também é destacada a motivação para o entretenimento e motivação para a aprendizagem nos jogos sérios;

- desafio - Conflito entre sistema e jogador(es), sendo Player VS Game (single player) e Player VS Player (multiplayer). O objetivo foi dividido em: Saúde ou cura quando existe um paciente e uma doença e o objetivo do jogador é identificar a doença e obter a cura para o paciente. Gestão faz referência a jogos cujo objetivo é ter sucesso no empreendimento hospitalar. Além destes, existem jogos em que o objetivo é zombar, ou seja fazer troça com os procedimentos médicos. O desafio pode ser incrementado com ameaças que foram divididas em ameaçar ao avatar que representa o jogador no jogo, ameaças ao paciente (e.g. paciente tem diabete, mas não sabia antes de começar o procedimento) e situacionais com o ambiente (e.g. acaba a luz);

- interação - Diferentemente do jogo cooperativo que a comunicação é fator determinante para a dinâmica cooperativa, neste modelo a comunicação não é tão relevante não sendo destacada na análise. A representação do jogador pode ser com avatares fixos ou customizáveis e suas habilidades podem ser iguais ou diferentes, fixas ou crescentes;

- processo do jogo - o estado do jogo foi compreendido como mundo aberto ou mundo fechado. O estado de espera nas pausas foi analisado pelos indicadores timer, idle, congelado e advertência. Os feedbacks foram divididos em informações sobre personagem, ameaça, tempo, situação. Os indicadores de feedback na interface foram identificados nas posições: superior, inferior, direita, esquerda e centro. Foi acrescentado o feedback pedagógico como elemento fundamental em jogos para a aprendizagem. Jogos com propósitos educativos devem apresentar ao jogador informações didáticas sobre seu desempenho, sendo, neste caso, necessário que o projeto de jogo contemple feedback visando o ensino-aprendizagem. O acréscimo de feedback e instrumentos avaliativos geram a possibilidade de o jogo oferecer uma avaliação pedagógica do desempenho do jogador;

- ações do jogador - neste quesito foi analisado as ações do jogador (físicas, táticas, de progressão e de interação social). Os objetos manipuláveis durante o jogo definidos como o próprio paciente e os objetos (props) que o jogador usa para realizar o procedimento;

- eventos do sistema - O sistema foi dividido em eventos, riscos e recompensas. Os eventos foram avaliados em termos do uso de randomização e progressão. Os riscos e recompensas foram analisados como fixos ou crescentes, sendo o aumentando randômico, constante e exponencial. Por fim a recompensa foi analisada em monetária (moeda de jogo), progressão de fase e bônus; 
Munhoz, D. R. M., Mazza, L. U., Taguchi, M. S., Spinillo, C. G., Fadel, L. M., Oliveira, A. E. F., Haddad, A. E., Rabêlo Jr., D. J. L. \& Rodrigues, A. P. M. G. | Análise de mecânicas de jogos e tendências gráficas na representação de jogos de procedimentos médicos, visando o estímulo e motivação para o aprendizado

- meio - foi acrescentada a análise do meio em que ocorre o jogo, se analógico ou digital nas diversas plataformas. E a utilização recursos de RV e RA (realidade virtual ou realidade aumentada). A RV e RA nos jogos digitais podem acrescentar agregar uma informação com função diferenciada ao jogo.

\section{Resultados}

O protocolo de análise adotado identifica a presença dos elementos de: motivação, desafios, interação, processo do jogo, ações do jogador e eventos do sistema. A conceituação do protocolo se baseou nos princípios de estética, mecânicas e dinâmica de jogo - o MDA (Hunicke, et al. 2004).

Sobe os jogos da amostra ( $n=10)$ : 6 eram de simulação de cirurgia, 2 de gestão hospitalar e 2 de atendimento clínico. Sendo apenas os jogos de atendimento clínico considerados jogos sérios desenvolvidos para propósito específico de aprendizagem, os outros são jogos desenvolvidos para entretenimento. Os jogos foram lançados no intervalo de 1988 a 2016. A coletada ocorreu nos sites de entretenimento (ojogos.com.br), plataformas de compra de jogos (Steam) e sites de crítica de jogos (IGN e Board Game Geek). Os resultados da análise são apresentados na tabela 5 e tabela 6.

Tabela 5: Síntese da análise de aspectos conceituais

\begin{tabular}{|c|c|c|c|c|c|c|c|c|c|c|c|c|c|}
\hline \multicolumn{3}{|c|}{ Aspectos conceituais } & 1 & 2 & 3 & 4 & 5 & 6 & 7 & 8 & 9 & 10 & Total \\
\hline \multirow[t]{5}{*}{ Motivo } & \multirow{3}{*}{$\begin{array}{l}\text { Tema / } \\
\text { Situação } \\
\text { imaginária }\end{array}$} & simulador de cirurgia & 1 & & & 1 & 1 & 1 & & & 1 & 1 & 6 \\
\hline & & gestão hospitalar & & 1 & 1 & & & & & & & & 2 \\
\hline & & atendimento clínico & & & & & & & 1 & 1 & & & 2 \\
\hline & \multirow[t]{2}{*}{ Propósito } & entretenimento & 1 & 1 & 1 & 1 & 1 & 1 & & & 1 & 1 & 8 \\
\hline & & aprendizagem & & & & & & & 1 & 1 & & & 8 \\
\hline \multirow[t]{8}{*}{ Desafio } & \multirow[t]{2}{*}{ Conflito } & player/s VS game & 1 & & 1 & 1 & 1 & 1 & & & 1 & 1 & 7 \\
\hline & & player VS player & & 1 & & & & & 1 & 1 & & & 3 \\
\hline & \multirow[t]{3}{*}{ Objetivo } & saúde ou cura & & & & 1 & 1 & & 1 & 1 & & 1 & 5 \\
\hline & & gestão & & 1 & 1 & & & 1 & & & & & 3 \\
\hline & & zombar & 1 & & & & & & & & 1 & & 1 \\
\hline & \multirow[t]{3}{*}{ Ameaças } & ao avatar do jogador & & & & & 1 & 1 & & & 1 & 1 & 4 \\
\hline & & ao paciente & 1 & 1 & 1 & 1 & 1 & 1 & & & 1 & 1 & 8 \\
\hline & & situacional & & 1 & 1 & & & & 1 & & 1 & & 4 \\
\hline \multirow[t]{6}{*}{ Interação } & \multirow{6}{*}{$\begin{array}{l}\text { Represen- } \\
\text { tação do } \\
\text { jogador }\end{array}$} & avatar fixo & 1 & 1 & 1 & 1 & 1 & 1 & 1 & 1 & 1 & 1 & 10 \\
\hline & & avatar customizável & & & & & & & & & & & 0 \\
\hline & & habilidades iguais & 1 & & & & & & 1 & 1 & 1 & & 4 \\
\hline & & habilidades diferentes & & 1 & 1 & 1 & 1 & 1 & & & & 1 & 6 \\
\hline & & habilidades fixas & 1 & 1 & 1 & 1 & 1 & 1 & 1 & 1 & 1 & 1 & 10 \\
\hline & & habilidades crescentes & & & & & & & & & & & 0 \\
\hline
\end{tabular}

Tabela 6: Síntese da análise de aspectos operacionais

\begin{tabular}{|c|c|c|c|c|c|c|c|c|c|c|c|c|c|}
\hline \multicolumn{3}{|c|}{ Aspectos operacionais } & 1 & 2 & 3 & 4 & 5 & 6 & 7 & 8 & 9 & 10 & Total \\
\hline \multirow{13}{*}{$\begin{array}{l}\text { Processo } \\
\text { do jogo }\end{array}$} & \multirow[t]{2}{*}{ Mundo } & fechado & 1 & 1 & 1 & 1 & 1 & 1 & 1 & & & 1 & 8 \\
\hline & & aberto & & & & & & & & & & & 0 \\
\hline & \multirow{4}{*}{$\begin{array}{l}\text { Estado de } \\
\text { espera nas } \\
\text { pausas }\end{array}$} & congelado & 1 & 1 & 1 & & 1 & 1 & 1 & 1 & 1 & 1 & 9 \\
\hline & & idle & & & & 1 & & & & & & & 1 \\
\hline & & timer & & & & & & & & & 1 & & 1 \\
\hline & & advertência & & & & & 1 & & & & & 1 & 2 \\
\hline & \multirow[t]{4}{*}{ Feedback } & personagem & & 1 & 1 & & 1 & & & & & 1 & 4 \\
\hline & & ameaça & 1 & 1 & 1 & 1 & 1 & 1 & 1 & & & 1 & 8 \\
\hline & & tempo & 1 & & & & 1 & 1 & & & 1 & 1 & 5 \\
\hline & & situação & 1 & 1 & 1 & 1 & & 1 & 1 & 1 & 1 & 1 & 9 \\
\hline & \multirow[t]{3}{*}{ Posição } & superior & 1 & & & 1 & 1 & 1 & & & 1 & 1 & 6 \\
\hline & & inferior & & & & & & & 1 & 1 & 1 & & 3 \\
\hline & & direita & & & & & & & & & 1 & & 1 \\
\hline
\end{tabular}


Munhoz, D. R. M., Mazza, L. U., Taguchi, M. S., Spinillo, C. G., Fadel, L. M., Oliveira, A. E. F., Haddad, A. E., Rabêlo Jr., D. J. L. \& Rodrigues, A. P. M. G. | Análise de mecânicas de jogos e tendências gráficas na representação de jogos de procedimentos médicos, visando o estímulo e motivação para o aprendizado

\begin{tabular}{|c|c|c|c|c|c|c|c|c|c|c|c|c|c|}
\hline & & esquerda & & & & & & 1 & & & 1 & & 1 \\
\hline & & centro & 1 & & & 1 & 1 & & 1 & 1 & 1 & 1 & 7 \\
\hline & \multicolumn{2}{|c|}{ Feedback pedagógico } & & & & & & & & & 1 & & 1 \\
\hline \multirow{7}{*}{$\begin{array}{l}\text { Ações do } \\
\text { jogador }\end{array}$} & \multirow[t]{5}{*}{ Mecânicas } & Física & 1 & 1 & 1 & 1 & 1 & 1 & 1 & & & 1 & 8 \\
\hline & & de economia & & & & & & & & & & & 0 \\
\hline & & de progressão & 1 & 1 & 1 & 1 & 1 & 1 & 1 & 1 & 1 & 1 & 10 \\
\hline & & táticas & & 1 & 1 & & & & 1 & & & & 3 \\
\hline & & de interação social & & & & & & & & & & & 0 \\
\hline & \multirow[t]{2}{*}{ Objetos } & paciente & 1 & 1 & 1 & 1 & 1 & 1 & 1 & & & 1 & 8 \\
\hline & & props & 1 & 1 & 1 & 1 & 1 & 1 & 1 & & & 1 & 0 \\
\hline \multirow{7}{*}{$\begin{array}{l}\text { Eventos } \\
\text { do } \\
\text { sistema }\end{array}$} & \multirow[t]{2}{*}{$\mathrm{IA}$} & randomizada & & & 1 & & & & 1 & & & & 2 \\
\hline & & progressão situacional & 1 & 1 & & 1 & 1 & 1 & & & 1 & 1 & 8 \\
\hline & \multirow[t]{2}{*}{ Risco } & riscos fixos & 1 & & & & & & 1 & 1 & 1 & & 4 \\
\hline & & riscos crescentes & & 1 & 1 & 1 & 1 & 1 & & & & 1 & 6 \\
\hline & \multirow[t]{3}{*}{$\begin{array}{l}\text { Recom- } \\
\text { pensa }\end{array}$} & $\begin{array}{l}\text { monetária } \\
\text { (moedas de jogo) }\end{array}$ & & & & & & 1 & & & & & 1 \\
\hline & & progressão & 1 & & 1 & 1 & 1 & 1 & & 1 & 1 & 1 & 8 \\
\hline & & bônus & 1 & 1 & 1 & & 1 & & & 1 & 1 & 1 & 7 \\
\hline \multirow[t]{6}{*}{ Meio } & \multirow[t]{4}{*}{ Plataforma } & celular & & & & & & & & 1 & & & 1 \\
\hline & & computador & 1 & & & 1 & & 1 & & & 1 & & 4 \\
\hline & & console & & & & & 1 & & & & 1 & 1 & 3 \\
\hline & & analógico & & 1 & 1 & & & & 1 & & & & 3 \\
\hline & \multirow[t]{2}{*}{ Recurso } & RV & & & & & & & & & 1 & & 1 \\
\hline & & RA & & & & & & & & & & & 0 \\
\hline
\end{tabular}

Nos aspectos conceituais houve a predominância de jogo de entretenimento $(n=8)$ e simulação de cirurgia $(n=6)$. O desafio principal foi pelo conflito player VS game $(n=7)$. Ameaças direcionadas ao paciente $(n=8)$. Jogador(es) representado(s) por avatar fixo $(n=10)$ com habilidades fixas $(n=10)$. Indicados no gráfico 1.

Nos aspectos conceituais foram menos presentes os jogos para aprendizagem - jogos sérios $(n=2)$ e situação de atendimento clínico $(n=2)$. O desafio menos comum foi do conflito player(s) VS player(s). Ameaças direcionadas ao avatar $(n=4)$. Jogador(es) representado(s) por avatar customizável $(n=0)$ com habilidades crescentes $(n=0)$. Indicados no gráfico 1.

Gráfico 1: Características conceituais mais e menos presentes

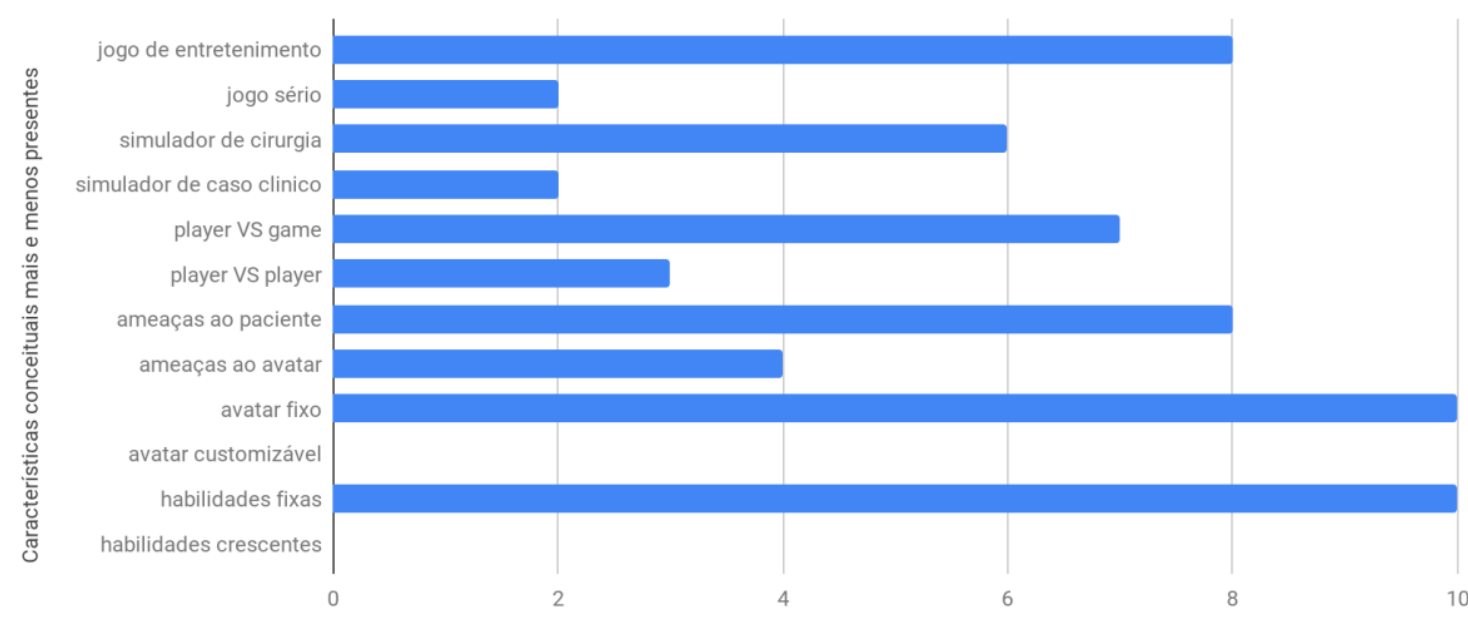

Nos aspectos operacionais a predominância foi de mundo fechado $(n=8)$. Estado de espera congelado $(n=7)$ nos momentos de pausa. Feedback mais comum foi de ameaças $(n=10)$. Na interface verificou-se os indicadores posicionados no centro $(n=5)$ na tela. As ações do jogador, a mecânica mais utilizada foi de progressão $(n=10)$. O sistema apresentou maior incidência de riscos fixos $(n=9)$ com aumento constante $(n=10)$. A principal recompensa foi a progressão 
Munhoz, D. R. M., Mazza, L. U., Taguchi, M. S., Spinillo, C. G., Fadel, L. M., Oliveira, A. E. F., Haddad, A. E., Rabêlo Jr., D. J. L. \& Rodrigues, A. P. M. G. | Análise de mecânicas de jogos e tendências gráficas na representação de jogos de procedimentos médicos, visando o estímulo e motivação para o aprendizado

( $n=8)$ e bônus ( $n=7)$. Indicados no gráfico 2.

Nos aspectos operacionais constatou-se a ausência de jogos de mundo aberto $(n=0)$. Estado de espera com indicador de timer $(n=1)$ e idle $(n=1)$ nos momentos de pausa. Baixa presença de feedback pedagógico $(n=1)$. Na interface verificou-se os indicadores posicionados na parte inferior $(n=1)$, direita $(n=1)$ e esquerda $(n=2)$ da tela. As ações do jogador sem presença de mecânicas de economia $(n=0)$. O sistema apresentou menor incidência de riscos crescentes $(n=0)$. Baixo uso de recursos de realidade virtual $(n=1)$ e ausência de realidade aumentada $(\mathrm{n}=0)$. Indicados no gráfico 2 .

Gráfico 2: Características operacionais mais e menos presentes

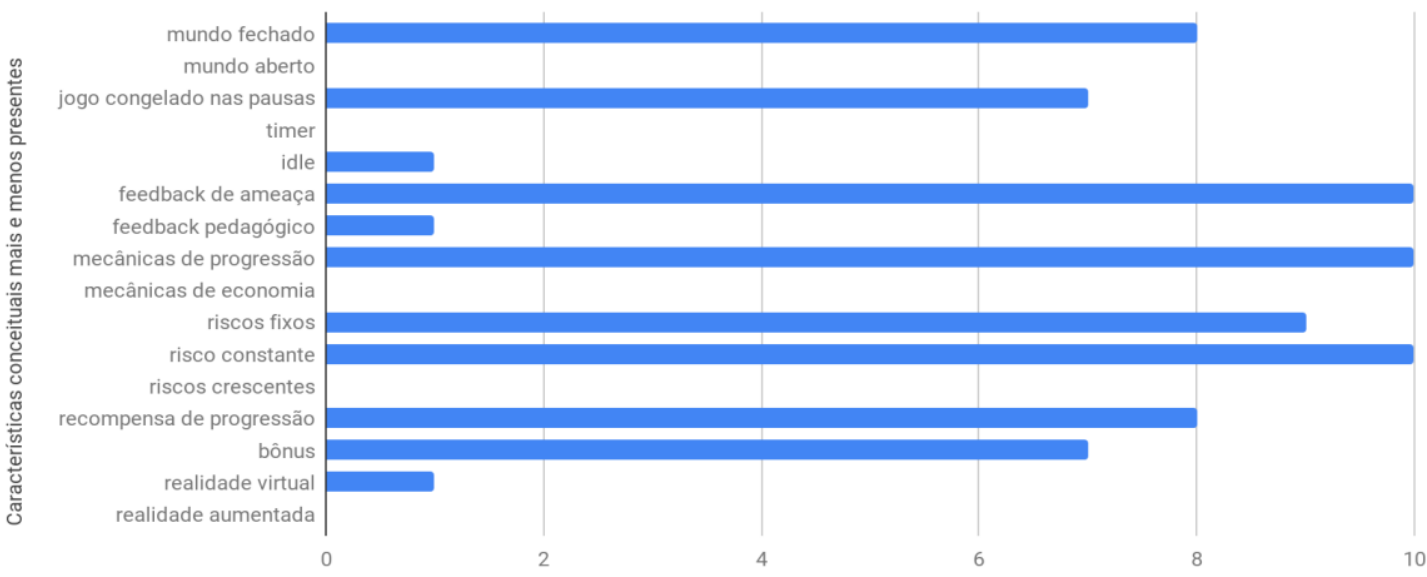

\section{Características de interface da amostra}

$\mathrm{Na}$ imagem 1 (Surgeon Simulator) pode ser visto indicador de tempo circulada em verde, e um exemplo de feedback textual em box no canto direito superior, circulado em rosa. Circulado em amarelo está um exemplo do jogador utilizando o paciente e o prop como objeto manipulável.

Imagem 1: Interface do jogo de entretenimento Surgeon Simulator

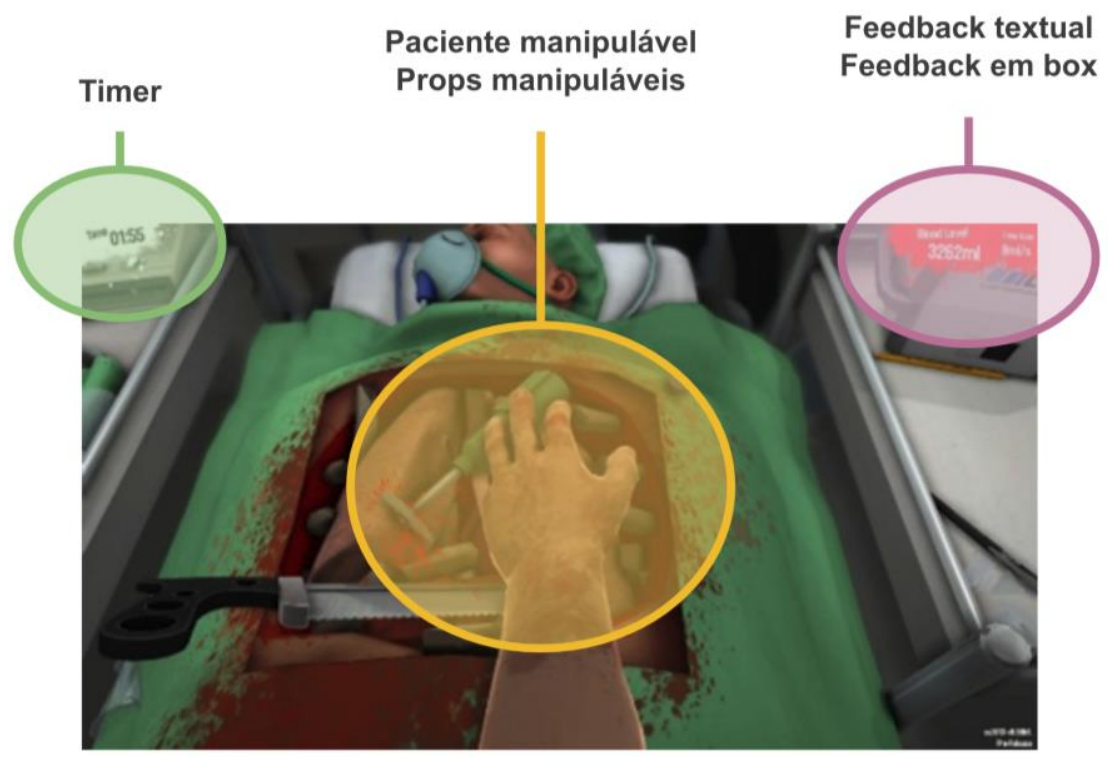

Anais do 9 Congresso Internacional de Design da Informação | CIDI 2019

Proceedings of the 9th Information Design International Conference 
Munhoz, D. R. M., Mazza, L. U., Taguchi, M. S., Spinillo, C. G., Fadel, L. M., Oliveira, A. E. F., Haddad, A. E., Rabêlo Jr., D. J. L. \& Rodrigues, A. P. M. G. | Análise de mecânicas de jogos e tendências gráficas na representação de jogos de procedimentos médicos, visando o estímulo e motivação para o aprendizado

$\mathrm{Na}$ imagem 2 (Life and Death), pode ser visto diversos tipos de feedbacks visuais circulados em verde. Circulado em amarelo está um exemplo de paciente e objetos como objeto manipulável. Em azul está um exemplo de feedback textual em box no canto direito inferior. Em rosa está apresentado o inventário.

Imagem 2: Interface do jogo de entretenimento Life and Death

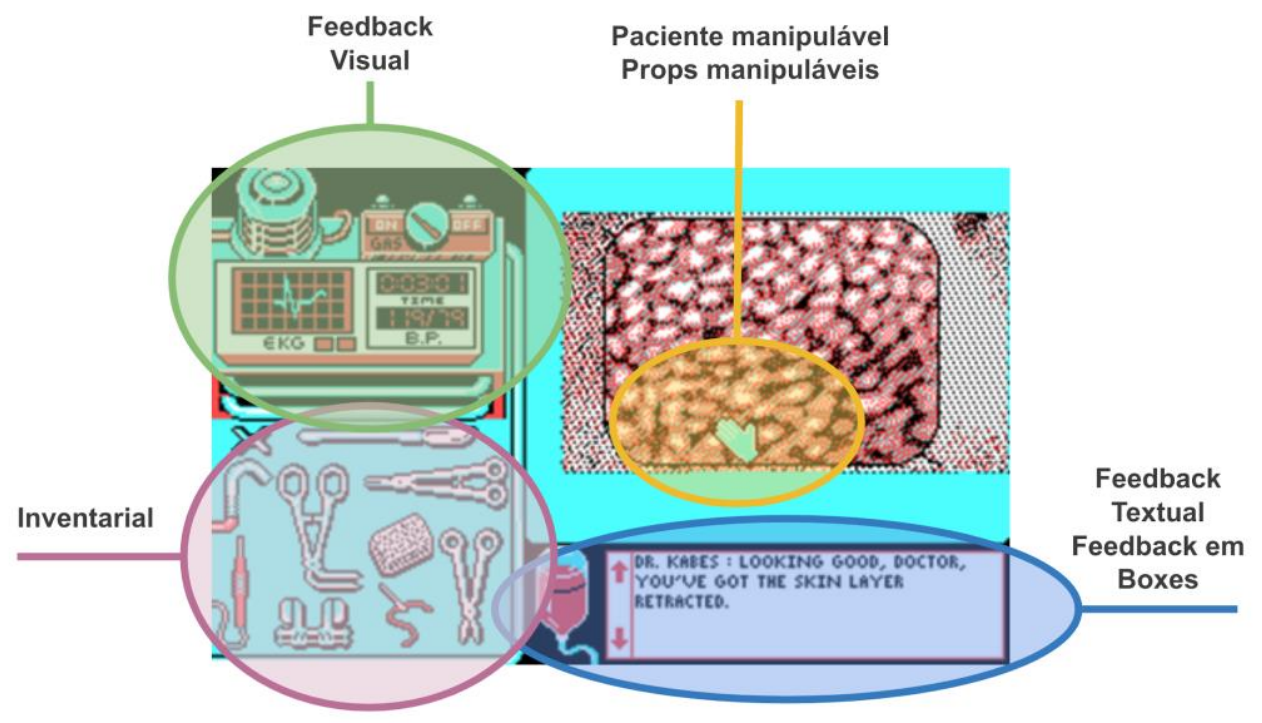

$\mathrm{Na}$ imagem 3 (Amateur Surgeon), está circulado em verde diferentes tipos de feedback, como timer, feedback textual e de cor. Em amarelo está apresentado o jogador utilizando props e o paciente como objeto manipulável. Em rosa está apresentado o inventário e em azul um exemplo de feedback textual.

Imagem 3: Interface do jogo de entretenimento Amateur Surgeon

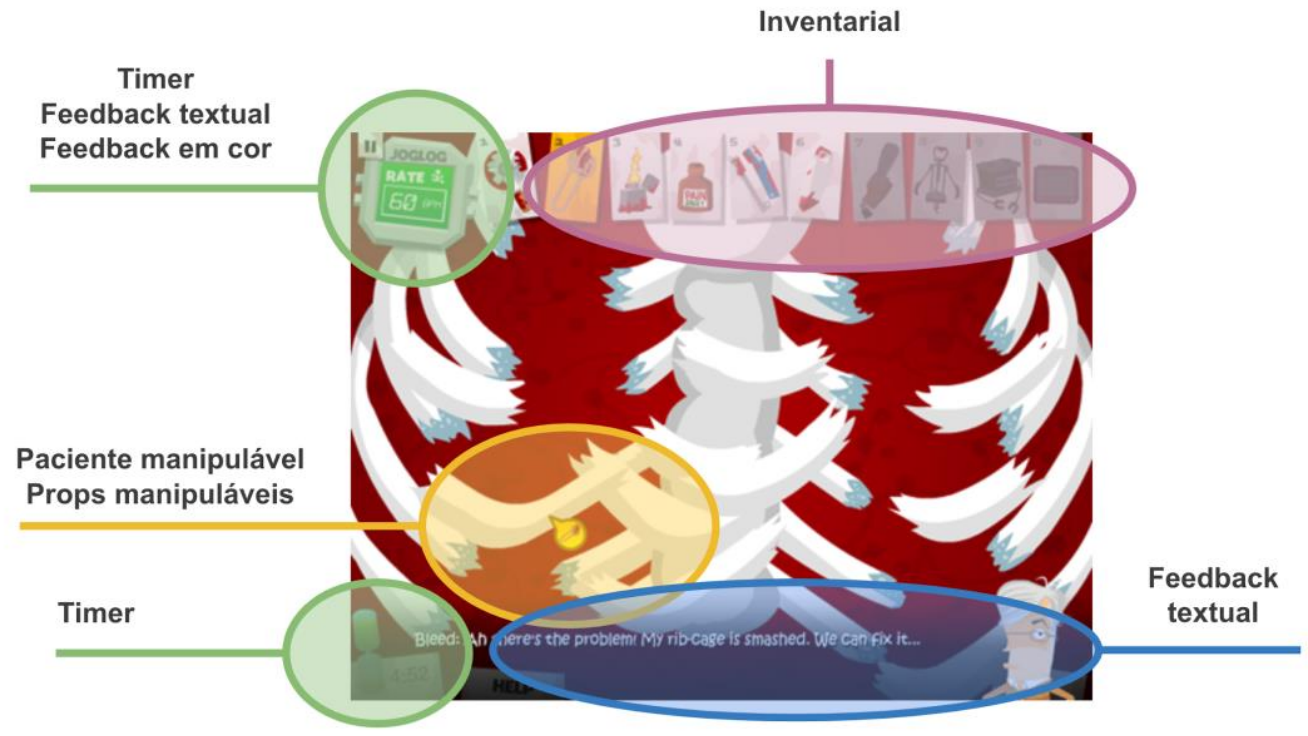

Anais do 9ํ Congresso Internacional de Design da Informação | CIDI 2019

Proceedings of the 9th Information Design International Conference 
Munhoz, D. R. M., Mazza, L. U., Taguchi, M. S., Spinillo, C. G., Fadel, L. M., Oliveira, A. E. F., Haddad, A. E., Rabêlo Jr., D. J. L. \& Rodrigues, A. P. M. G. | Análise de mecânicas de jogos e tendências gráficas na representação de jogos de procedimentos médicos, visando o estímulo e motivação para o aprendizado

\section{Conclusão e desdobramentos}

Os jogos analisados para procedimentos médicos apresentaram simulação de procedimentos médicos com narrativa predominantemente linear, onde as escolhas feitas pelos jogadores não alteram o rumo da história, todos os jogos analisados eram ambientados em mundos fechados.

A representação dos jogadores foi limitada a avatares fixos sem opções de customização dos personagens. Os riscos ou desafios do jogo são, na maioria, fixos e a progressão é linear com possibilidade de intervenção centrada no paciente. A amostra mostrou baixos índices de oportunidades de escolha, resultando em uma árvore de possibilidades limitada, características que diminuem os índices de re-jogabilidade (Brathwaite \& Schreiber, 2009) podendo reduzir a motivação do jogador. O desequilíbrio entre o desafio e a dificuldade pode vir a causar a frustração e não oferecer estímulos para a aprendizagem (Csikszentmihalyi, 1990).

A identificação destas características indica que existe uma lacuna para jogos de procedimentos médicos em mundo aberto com distribuição de papéis e customização de avatares. Jogos RPG multiplayer podem contar com a interação entre os jogadores permitindo ampliar a dinâmica do jogo. Identificando acima de tudo a necessidade de leque de possibilidades amplo exigindo maior esforço do jogador na tomada de decisão, situação que pode levar ao fenômeno de aprendizado pela descoberta (Betrancourt, 2005) em jogos que contemplem um espaço para o jogador refletir e construir suas próprias conclusões a partir dos fatos expostos e desenvolver seu aprendizado. A amostra identificou baixa exploração do uso de recursos de Realidade Aumentada, recursos que pode ser instrumento para ampliar as informações fornecidas ao jogador. E, principalmente, a necessidade de se inserir presença de feedback didático para transformar a experiência de jogo em uma atividade didática.

\section{Agradecimento}

A UNASUS-UFMA pela abertura de espaço para o desenvolvimento de sistemas de jogo para aprendizagem de conteúdos de saúde para EaD.

\section{Referências}

Barnes, S., \& Prescott, J. (2018). Empirical evidence for the outcomes of therapeutic video games for adolescents with anxiety disorders: systematic review. JMIR serious games, $6(1)$, e3.

Betrancourt, M. (2005). The animation and interactivity principles in multimedia learning. The Cambridge handbook of multimedia learning, 287-296.

Brathwaite, B., \& Schreiber, I. (2009). Challenges for game designers. Nelson Education.

Castel, A. D., Qasmieh, S., Greenberg, D., Ellenberger, N., Howell, T. H., Griffith, C., ... \& Rakhmanina, N. (2018). Digital gaming to improve adherence among adolescents and young adults living with HIV: mixed-methods study to test feasibility and acceptability. JMIR serious games, 6(4), e10213.

Csikszentmihalyi, M. (1990). Flow: The psychology of optimal experience. New York: Harper \& Row.

Dormans, J. (2012). Engineering emergence: applied theory for game design. Universiteit van Amsterdam [Host].

Garris, R., Ahlers, R., \& Driskell, J. E. (2002). Games, motivation, and learning: A research and practice model. Simulation \& gaming, 33(4), 441-467.

Hunicke, R., LeBlanc, M., \& Zubek, R. (2004, July). MDA: A formal approach to game design and game research. In Proceedings of the AAAI Workshop on Challenges in Game Al (Vol. 4, No. 1, p. 1722). 
Munhoz, D. R. M., Mazza, L. U., Taguchi, M. S., Spinillo, C. G., Fadel, L. M., Oliveira, A. E. F., Haddad, A. E., Rabêlo Jr., D. J. L. \& Rodrigues, A. P. M. G. | Análise de mecânicas de jogos e tendências gráficas na representação de jogos de procedimentos médicos, visando o estímulo e motivação para o aprendizado

Munhoz, Daniella R. M. (2018). Design de jogos de tabuleiro e dinâmicas cooperativas: uma abordagem histórico-cultural. Tese (Doutorado em Design) - Setor de Artes, Design e Comunicação, Universidade Federal do Paraná.

Michael, D. R., \& Chen, S. L. (2005). Serious games: Games that educate, train, and inform. Muska \& Lipman/Premier-Trade.

Rodriguez, H. (2006). The playful and the serious: An approximation to Huizinga's Homo Ludens. Game Studies, 6(1), 1604-7982.

Salen, K., Tekinbaş, K. S., \& Zimmerman, E. (2004). Rules of play: Game design fundamentals. MIT press.

Susi, T., Johannesson, M., \& Backlund, P. (2007). Serious games: An overview.

UNA-SUS/UFMA Site http://www.unasus.ufma.br/site/una-sus-ufma/sobre Acessado pela última vez no dia 07/07/2019, 15:45.

Willmott, T., Russell-Bennett, R., Drennan, J., \& Rundle-Thiele, S. (2019). The impact of serious educational gameplay on adolescent binge drinking intentions: a theoretically grounded empirical examination. Health Education \& Behavior, 46(1), 114-125.

\section{Sobre o(a/s) autor(a/es)}

Daniella Rosito Michelena Munhoz, PhD, UFPR, Brasil <daniella.ufpr@gmail.com>

Larissa Ugaya Mazza, UFPR, Brasil <Lari.U.Ma@gmail.com>

Marcelo Satoshi Taguchi, UFPR, Brasil <staguchi.br@gmail.com>

Carla Galvão Spinillo, PhD, UFPR, Brasil <cgspin@gmail.com>

Luciane Fadel, PhD, UFSC, Brasil <liefadel@gmail.com>

Ana Emilia Figueiredo Oliveira, PhD, UFMA <oliveira.anaemilia@gmail.com>

Ana Estela Haddad, PhD, USP <aehaddad@usp.br>

Dilson José Lins Rabêlo Júnior, USASUS, Brasil <dilsonrabelo.unasus@gmail.com>

Alan Palomero Machado Góes Rodrigues, UFPR, Brasil <alan.palomero@gmail.com> 